\title{
EFEITO DO INTERVALO ENTRE SESSÕES DE EXERCÍCIO DE FORÇA SOBRE O DESEMPENHO NEUROMUSCULAR
}

\author{
EFFECT OF INTERVAL BETWEEN STRENGTH EXERCISE SESSIONS ON NEUROMUSCULAR PERFORMANCE
}

Artigo Original

\author{
EFECTO DEL INTERVALO ENTRE SESIONES DE EJERCICIO DE FUERZA SOBRE EL
}

DESEMPENO NEUROMUSCULAR

\author{
Charles Ricardo Lopes ${ }^{1,2}$ \\ (Educador Físico) \\ Alex Harley Crisp ${ }^{1,5}$ \\ (Educador Físico) \\ Marcio Antonio Gonsalves Sindorf' \\ (Educador Físico) \\ Moises Diego Germano \\ (Educador Físico) \\ Luís Guilherme Lutgens \\ (Educador Físico) \\ Camila Amorim Nardin' \\ (Educador Físico) \\ Gustavo Ribeiro da $\mathrm{Mota}^{3}$ \\ (Educador Físico) \\ Marcelo Saldanha Aoki ${ }^{4}$ \\ (Educador Físico e Nutricionista) \\ Rozangela Verlengia' (Biólogo)
}

1. Grupo de Pesquisa em Ciências da Performance Humana, Universidade Metodista de Piracicaba (UNIMEP), Piracicaba, SP, Brasil.

2. Faculdade Adventista de Hortolândia, Hortolândia (UNASP), São Paulo, SP, Brasil.

3. Universidade Federal do

Triângulo Mineiro (UFTM),

Uberaba, MG, Brasil.

4. Escola de Artes, Ciências e

Humanidades, Universidade de São

Paulo (USP), São Paulo, SP, Brasil.

5. Universidade Estadual Paulista

(UNESP), Araraquara, SP, Brasil.

\section{Correspondência:}

Universidade Metodista de Piracicaba (UNIMEP) - Campus Taquaral. Rodovia do Açúcar, km 156, s/n, Piracicaba, SP, Brasil. 13400-911.chrlopes@unimep.br

\section{RESUMO}

Introdução: O intervalo de recuperação entre sessões é uma variável do treinamento de força fundamental para garantir a recuperação da capacidade neuromuscular. Objetivo: Comparar o efeito do tempo de recuperação entre sessões consecutivas de exercício de força sobre o desempenho neuromuscular de homens treinados. Métodos: Oito homens treinados em força ( $21,6 \pm 3,3$ anos; $75,1 \pm 11,3 \mathrm{~kg} ; 178,2 \pm 6,8 \mathrm{~cm})$ realizaram duas sessões de exercício de força randomizadas e cruzadas, repetidas com intervalo de recuperação de $24 \mathrm{~h}$ ou $48 \mathrm{~h}$. As sessões consistiram dos exercícios supinos reto, inclinado e declinado executadas em cinco séries de 10 repetições com intensidade de 70\% de uma repetição máxima (1RM) para cada exercício. O desempenho neuromuscular pós-sessão de exercício (força, potência e velocidade), foi avaliado por meio de acelerômetro (Myotest ${ }^{\circledR}$ ), com carga de 50\% de 1RM, no exercício supino reto. Resultados: Ambas as sessões (24 e 48h) apresentaram redução significante $(P<0,05)$ na capacidade neuromuscular (força, potência e velocidade) pós-sessão de treinamento, apresentando retorno ao valor pré no intervalo de $24 \mathrm{~h}(P>0,05)$. Conclusão: Os resultados sugerem que o intervalo de recuperação de 24 h é suficiente para a recuperação do desempenho neuromuscular de membros superiores em homens treinados.

Palavras-chave: força muscular, teste de esforço, treinamento de resistência.

\section{ABSTRACT}

Introduction: The rest interval between bouts is a crucial variable of resistance training to ensure recovery of neuromuscular capacity. Objective: To compare the effect of rest interval between repeated bouts of resistance training on neuromuscular capacity of trained men. Methods: Eight resistance-trained men (21.6 \pm 3.3 years, $75.1 \pm 11.3 \mathrm{~kg}, 178.2 \pm$ $6.8 \mathrm{~cm}$ ) performed two randomized and crossover resistance exercise bouts, repeated with rest interval of $24 \mathrm{~h}$ or $48 \mathrm{~h}$. The bouts consisted of horizontal, inclined and declined bench press performed with five sets of 10 repetitions with an intensity of $70 \%$ of one repetition maximum (1RM) for each exercise. Neuromuscular performance post-exercise bout (strength, power and speed), was assessed with an accelerometer (Myotes $\left.{ }^{\circledR}\right)$, with a load of 50\% 1RM, in the bench press exercise. Results: Both sessions (24 and 48h) showed significant reduction ( $P<0.05)$ in the neuromuscular capacity (strength, power and speed) post-exercise bout, returning to baseline values within $24 h$ ( $P>0.05)$. Conclusion: The results suggest that the rest interval of $24 \mathrm{~h}$ is sufficient for recovery of neuromuscular performance in upper limbs of resistance-trained men.

Keywords: muscle strength, exercise test, resistance training.

\section{RESUMEN}

Introducción: El intervalo de recuperación entre sesiones es una variable del entrenamiento de fuerza fundamental para garantizar la recuperación de la capacidad neuromuscular. Objetivo: Comparar el efecto del tiempo de recuperación entre sesiones consecutivas de entrenamiento de la fuerza en el rendimiento neuromuscular de hombres entrenados. Métodos: Ocho hombres entrenados en fuerza (21,6 $\pm 3,3$ años y 75,1 $\pm 11,3 \mathrm{~kg}, 178,2 \pm 6,8$ $\mathrm{cm}$ ) realizaron dos sesiones de ejercicios aleatorios y cruzados, repetidas a intervalos de fuerza $24 \mathrm{~h}$ o $48 \mathrm{~h}$ de recuperación. Las sesiones consistieron en ejercicios supinos recto, inclinado y declinado siendo ejecutadas cinco series de 10 repeticiones con una intensidad de $70 \%$ de una repetición máxima (1RM) para cada ejercicio. El rendimiento neuromuscular después de la sesión de ejercicio (fuerza, potencia y velocidad), fue evaluado por medio de acelerómetro (Myotest $\left.{ }^{\circledR}\right)$, con carga de 50\% de IRM en el ejercicio supino recto. Resultados: Ambas sesiones (24 y $48 \mathrm{~h}$ ) mostraron una reducción significativa $(P<0,05)$ en la capacidad neuromuscular (fuerza, potencia y velocidad) después de la sesión de entrenamiento, presentando retorno al valor anterior en el intervalo de $24 \mathrm{~h}(P>0,05)$. Conclusión: Los resultados sugieren que el intervalo de recuperación de $24 \mathrm{~h}$ es suficiente para la recuperación del desempeño neuromuscular de miembros superiores en hombres entrenados.

Palabras clave: fuerza muscular, prueba de esfuerzo, entrenamiento de resistencia. 


\section{INTRODUÇÃO}

O treinamento de força (TF) é, indubitavelmente, uma das estratégias mais populares de treinamento físico. Entretanto, o sucesso do programa de TF para promover as adaptações desejadas depende da complexa interação de diversas variáveis (intensidade, volume, pausa, velocidade e tipo da ação muscular e a frequência)' . Dentre essas, a frequência semanal das sessões de treinamento é um componente fundamental, uma vez que o mesmo determina o número de estímulos e o intervalo de recuperação.

O Colégio Americano de Medicina do Esporte $^{2}$ preconiza que indivíduos iniciantes deveriam utilizar uma frequência de duas a três sessões na semana. Já indivíduos intermediários deveriam atingir a frequência de três a quatro sessões por semana, enquanto que indivíduos avançados deveriam aumentar o número de estímulos para 4 a 5 sessões por semana. Assim, a partir dessas recomendações é possível estimar o tempo de recuperação entre as sessões. Para iniciantes, por exemplo, o intervalo entre as sessões seria superior a 48 horas, podendo ser maior ainda, para algumas sessões na semana. Já para indivíduos avançados, o intervalo poderia ser reduzido em 24 horas para algumas sessões de treinamento.

É sabido que o aumento abrupto e persistente da carga de treinamento, sem efetiva recuperação entre sessões de treinamento, pode resultar na perda acentuada do desempenho neuromuscular ou mesmo iniciar um estado de mal-adaptação ${ }^{3,4}$. Embora, o intervalo de recuperação entre sessões seja determinante das respostas ao TF, essa variável foi pouco investigada ${ }^{5-7}$, e não existe consenso com relação ao melhor intervalo de recuperação entre as sessões ${ }^{8}$.

Vários são os mecanismos envolvidos no processo de recuperação entre sessões de exercício de alta intensidade, entre estes: a recomposição energética, principalmente dos estoques de glicogênio muscular ${ }^{9}$ e regeneração do dano muscular ${ }^{10}$. Com relação ao dano, durante o processo de treinamento o efeito da sessão repetida (repeated bout effect) está envolvido na diminuição da magnitude do dano muscular para a realização das sessões de exercícios subsequentes ${ }^{11}$. Tais processos são intensificados ao longo do tempo de treinamento, diminuindo os sintomas provenientes do dano muscular, como a percepção de dor muscular de início tardio, desempenho neuromuscular e entre outros. Desta forma, a magnitude do dano muscular frente à realização de uma sessão de alta intensidade e período de recuperação entre indivíduos treinados e sedentários são diferentes ${ }^{12}$.

Estudos a respeito da influência da manipulação das variáveis do treinamento sobre a recuperação do desempenho neuromuscular ${ }^{5-7,13-16}$, contribuem com a prescrição e elaboração dos períodos de recuperação no processo de treinamento para a população treinada. Contudo, há lacuna de estudos que investigam a influência do intervalo de recuperação entre sessões em homens treinados em força.

O objetivo do presente estudo foi analisar o efeito de diferentes intervalos de recuperação ( 24 e 48 h) entre sessões repetidas de força ao longo do tempo pós-sessão de exercício em homens treinados. Nossa hipótese inicial foi de que o intervalo de 24 h seria insuficiente para a recuperação da capacidade neuromuscular, pós-sessão de treinamento de força.

\section{METODOLOGIA}

Participaram do presente estudo oito homens com (média \pm desvio

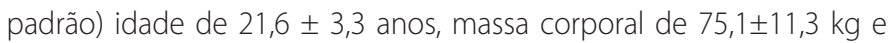
estatura de $178,2 \pm 6,8 \mathrm{~cm}$. Todos os voluntários estavam realizando rotinas diárias de exercício de força e tinham experiência de 4,8 2,2 anos no treinamento de força. Os participantes foram informados sobre a proposta do estudo, assinaram Termo de Consentimento Livre e Esclarecido e responderam questionário de avaliação de saúde. O referido estudo foi aprovado pelo Comitê de Ética em Pesquisa local e aprovado sob o número de protocolo 41/12.

Os critérios de inclusão para participação do estudo foram: (a) ter no mínimo de um ano de experiência no treinamento de força; (b) realizar sessões de exercício de força com frequência maior ou igual a quatro vezes por semana; (c) ser familiarizado no exercício supino e com teste de uma repetição máxima (1RM). Os critérios de exclusão foram: (a) não ter sofrido nenhuma lesão que possa interferir no estudo; (b) não estar utilizando suplementos nutricionais à base de creatina; (c) não fazer uso de esteroides anabolizantes.

Uma semana antes da realização das sessões experimentais de exercícios de força, foram realizados três encontros com intervalo de $24 \mathrm{~h}$ entre os mesmos, para a realização do teste de uma repetição máxima (1RM) para os exercícios: supino reto; supino inclinado e supino declinado. Após a determinação do desempenho neuromuscular a 50\% de 1RM em situação basal, todos voluntários $(n=08$ ) realizaram aleatoriamente e de maneira cruzada dois protocolos similares (volume, intensidade, pausa, velocidade de execução, sequência de exercícios) sendo duas sessões em cada protocolo, com diferenças apenas no tempo entre a primeira e a segunda sessão. Os protocolos foram denominados de: $24 \mathrm{~h}$ de intervalo de recuperação (P24) e 48h de intervalo de recuperação (P48) entre as duas sessões realizadas em cada protocolo. Dos 8 participantes, 4 realizavam $\mathrm{P} 24$ no primeiro momento e 4 realizaram P48. Após uma semana de intervalo, os grupos foram invertidos, caracterizando assim, um desenho cruzado balanceado (crossover).

Este trabalho é de natureza descritiva e transversal, todas as avaliações e procedimentos experimentais foram realizados sempre pelos os mesmos pesquisadores envolvidos no projeto. Para excluir qualquer efeito residual de exercício prévio sobre o tempo em que foi realizada a pesquisa, os participantes foram orientados a somente manter atividades da vida diária, sem a prática de outros exercícios físicos. $\mathrm{O}$ desempenho neuromuscular foi avaliado nos momentos: pré (primeira sessão), pós (primeira sessão), pré (segunda sessão), pós (segunda sessão), 24h e 48h para grupo P24 e pré (antes), pós (primeira sessão), 24h, pré (segunda sessão), pós (segunda sessão) 24h e 48h para o grupo P48.

A sessão de exercício de força consistiu na realização de cinco séries de 10 repetições com intensidade de 70\% de 1RM, com 1 minuto de pausa passiva entre as séries e 2 minutos entre exercícios. A ordem dos exercícios foi: supino reto, supino inclinado e supino declinado. Para garantir que o volume total de carga (séries $x$ repetições $x$ carga [kg]) fosse semelhante em ambas às condições (P24 e P48), os voluntários recebiam auxilio do pesquisador para completar os números de repetições estipulados em cada série, se necessário. As quatro sessões de exercícios (duas de cada protocolo - P24 e P48) foram realizadas entre 18h00min e 22h00min.

A determinação da força muscular máxima voluntária dinâmica para a prescrição da intensidade das sessões de exercício de força foi realizada pelo teste de 1RM, com intervalo de 24 horas entre os exercícios (supino reto, supino inclinado e supino declinado) de acordo com os procedimentos descritos por Brown e Weir ${ }^{17}$. Resumidamente, os voluntários realizaram um aquecimento de 2 a 3 séries de 5 a 10 repetições com aproximadamente 40-60\% 1RM estimado, antes da execução do protocolo. Posteriormente, os voluntários foram instruídos a realizar uma única repetição máxima e, a carga era ajustada aproximadamente em $\pm 10 \%$ para as tentativas subsequentes, até o voluntário conseguir concluir a tentativa com a técnica adequada do exercício. A determinação da força muscular máxima foi realizada com número máximo de cinco tentativas e intervalos de 3-5 minutos de recuperação entre essas.A determinação do desempenho neuromuscular foi realizada por meio de acelerômetro (Myotest ${ }^{\circledR}$ [MYO] - Myotest Inc, Suíça), no exercício supino reto seguindo os mesmo procedimentos descritos por Koshida et al. ${ }^{18}$. Resumidamente, o dispositivo foi posicionado na barra, entre o ombro e o polegar da mão direita, e mantido na posição vertical. Utilizando $50 \%$ do teste de 1 RM os voluntários foram instruídos a segurar a barra aproximadamente $2-3 \mathrm{~cm}$ acima do nível do peitoral, como posição inicial do movimento. Depois de um sinal sonoro emitido pelo acelerômetro, os voluntários empurravam a barra para cima em sua velocidade máxima até a completa extensão do cotovelo. Cada voluntário realizou três tentativas com pausa de 3 minutos entre as tentativas. 


\section{Análise Estatística}

A normalidade dos dados foi avaliada pelo teste Shapiro-Wilk. Em seguida, foi realizada análise de variância de um fator (ANOVA - One Way) com medidas repetidas, para a comparação da cinética das avaliações entre diferentes intervalos. O teste post hoc empregado foi Turkey. $O$ índice de significância adotado foi $P \leq 0.05$. Os dados estão expressos pela média \pm erro padrão da média (EPM).

\section{RESULTADOS}

Não foi observada diferença significante $(P>0.05)$ para o volume total de carga levantada entre as sessões de exercício (primeira sessão P24 = 8364.6 \pm 421.3 kg; segunda sessão P24 = 8422.9 \pm 439.2 kg; primeira sessão P48 = 8379.2 \pm 419.3 kg; segunda sessão P48 = 8366.7 \pm 421.1 kg).

Os dados relativos ao desempenho neuromuscular estão expressos nas figuras 1, 2 e 3. Em relação ao momento pós-primeira sessão de exercício (S1), os dados indicaram redução significativa $(P<0,001)$ para o grupo P24h nas variáveis: força (13\%), velocidade (49\%) e potência (53\%), quando comparado com o valor pré. Para o grupo P48h também foi observado redução significativa $(P<0,001)$ nas variáveis: força $(10 \%)$ velocidade (39\%) e potência (46\%).

Pós-segunda sessão de exercício (S2 - repetição), foi observado queda significativa $(P<0,001)$ para o grupo P24h (força 12\%; velocidade 45\% e potência 49\%) e para o grupo P48h (força 11\%; velocidade 39\% e potência 44\%), em comparação ao valor pré. No entanto, as variáveis de força, velocidade e potência retornaram aos valores pré $(P>0,05)$, no período de 24 h após a realização da primeira e segunda sessão de exercício de força. Quando comparados os valores entre sessões (P24h e P48h), não foi encontrada diferença significativa $(P>0,05)$ nos períodos: pré, pós, 24 e 48 h.

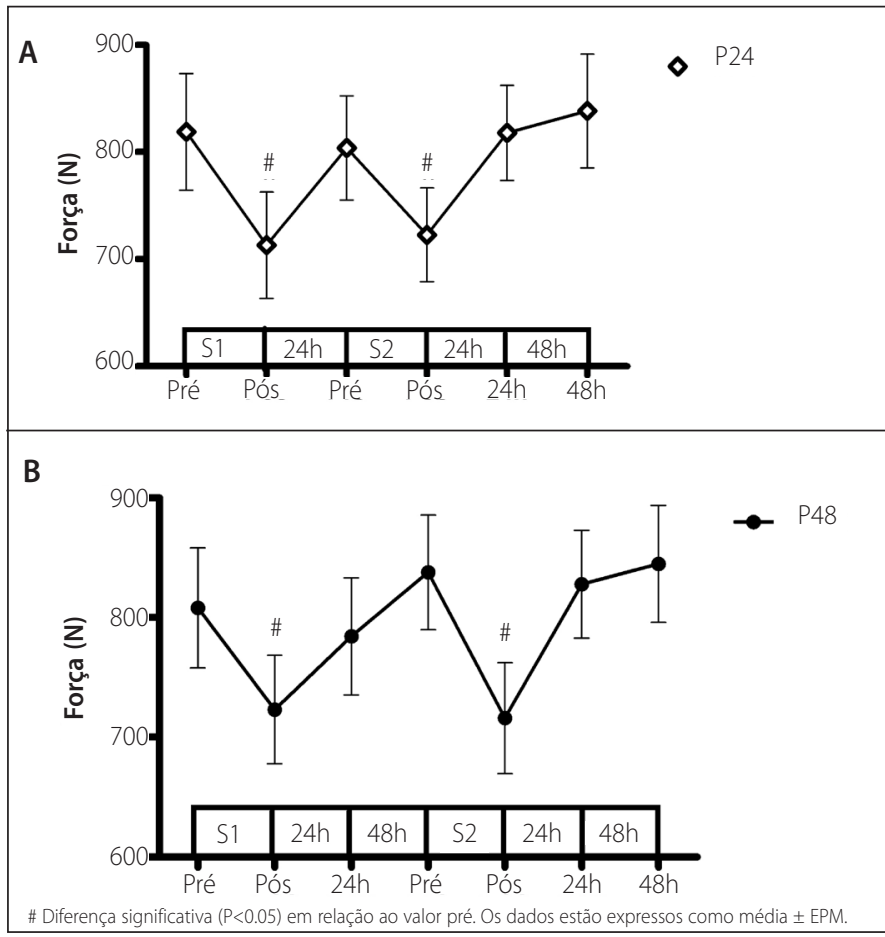

Figura 1. Valores de força (Newton) para o grupo P24 (A) e para o grupo P48 (B) no exercício supino reto ao longo do tempo pós-sessão de exercício de força. $\mathrm{S1}$ = primeira sessão de exercício; \$2 = segunda sessão de exercício.

\section{DISCUSSÃO}

Nossos principais achados indicam que ambos os intervalos (24 e 48h) foram suficientes para promover a recuperação da capacidade neuromuscular (força, velocidade e potência). Além disso, ambos os períodos de recuperação permitiram a plena realização da segunda sessão de treinamento nas mesmas condições de intensidade e volume da primeira, uma vez que

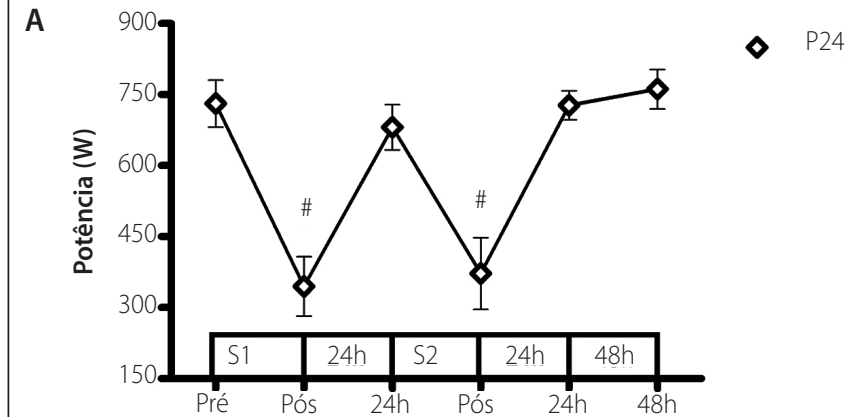

B

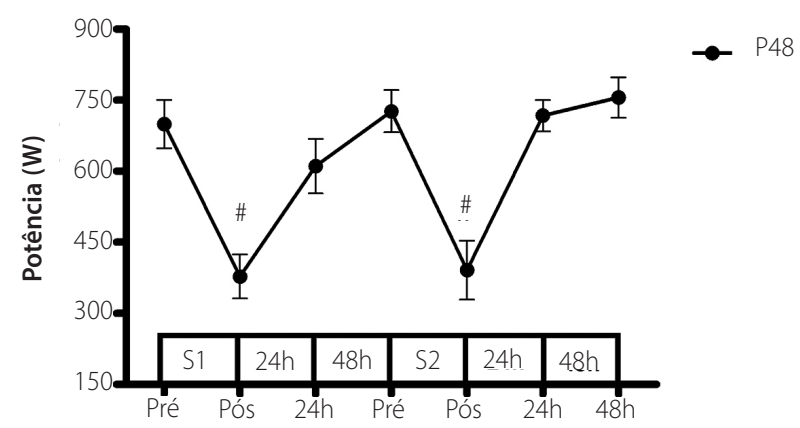

\# Diferença significativa $(P<0.05)$ em relação ao valor pré. Os dados estão expressos como média \pm EPM.

Figura 2. Valores de potência (Watts) para o grupo P24 (A) e para o grupo P48 (B) no exercício supino reto ao longo do tempo pós-sessão de exercício de força. $\mathrm{S1}$ = primeira sessão de exercício; $\$ 2$ = segunda sessão de exercício.

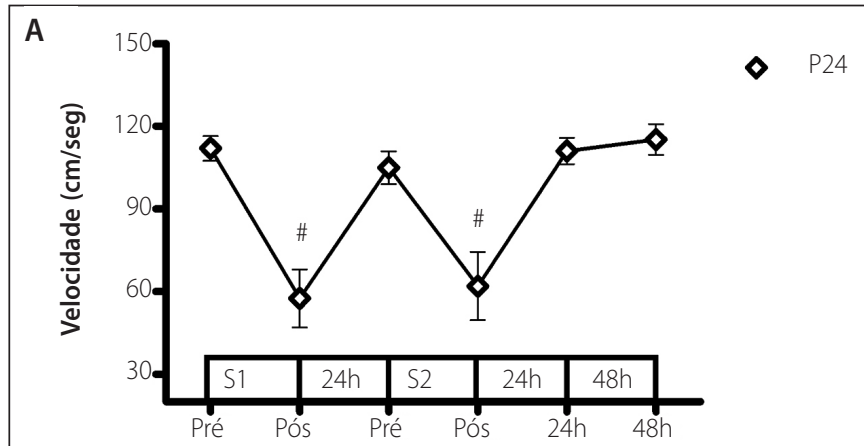

B

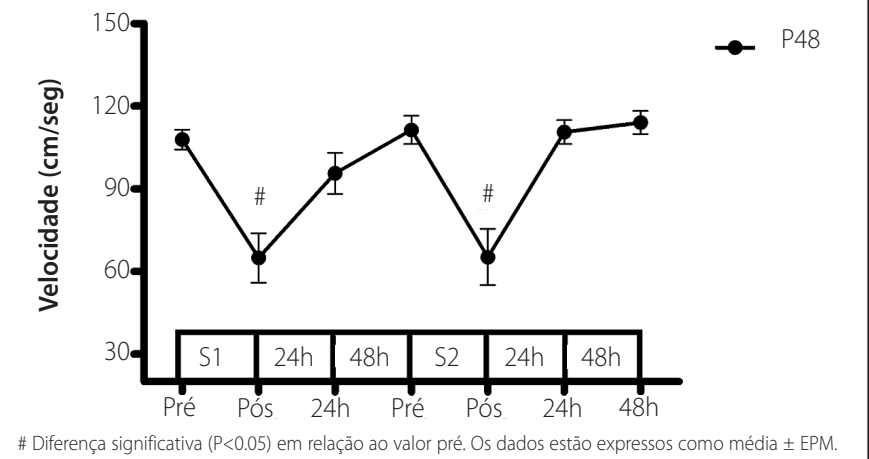

Figura 3. Valores de velocidade (centímetros por segundo) para o grupo P24 (A) e para o grupo P48 (B) no exercício supino reto ao longo do tempo pós-sessão de exercício de força. S1 = primeira sessão de exercício; $\mathbf{S 2}$ = segunda sessão de exercício.

o volume total entre as sessões para as duas condições de intervalo de recuperação (24 e 48h) foi mantido.

Os indivíduos treinados, avaliados no presente estudo, apresentaram queda significante do desempenho neuromuscular (força, velocidade e potência) no momento pós-sessão de exercício, provavelmente, resultado da fadiga aguda induzida pelo alto volume de trabalho mecânico 
(total de 150 repetições), intensidade (70\% 1RM) e curto intervalo de recuperação entre séries (1 minuto). Por outro lado, o desempenho neuromuscular retornou aos valores pré-exercício no período de $24 \mathrm{~h}$, assim, não necessitando de intervalo de recuperação maior que 48 horas.

Estes dados podem, em parte, ser explicados, pelas adaptações provenientes ao efeito da sessão repetida estarem presentes de forma significativa em indivíduos treinados ${ }^{19}$. Assim, devido à prática diária de treinamento de força, os voluntários estavam bem adaptados, fato que possivelmente, resultou em atenuação da magnitude do dano muscular e favoreceu a recuperação efetiva dos parâmetros de força, velocidade e potência em curto período de tempo (24 h), apesar da fadiga muscular observada no momento pós-primeira e segunda sessão de exercício.

Na literatura, observa-se valores distintos do tempo de recuperação do desempenho neuromuscular pós-sessão de exercício de força em homens treinados, com estudos reportando intervalos de $3 \mathrm{~h}^{14}, 24 \mathrm{~h}^{13,16}, 33 \mathrm{~h}^{14}, 48 \mathrm{~h}^{5-}$ $7,15,16$ e $72 \mathrm{~h}^{15}$. Certamente, a diferença na manipulação dos protocolos de treinamento entre os estudos (intensidade, volume, pausa entre séries, grupamento muscular envolvido, escolha e ordem dos exercícios e entre outros), contribui para a discrepância dos valores encontrados entres estudos.

No entanto, nossos resultados diferem dos obtidos por Mclester et al. 5 e Judge e Burke et al. ${ }^{8}$, os quais observaram recuperação do desempenho neuromuscular 48 horas pós-sessão envolvendo o exercício supino em homens treinados. Em adição, Lopes et al.,16 com objetivo de avaliar a influência de diferentes velocidades de execução (1,5 segundos vs 6 segundos) em uma sessão de treinamento de força (supino reto e inclinado: 5 séries de 12 repetições máximas), observaram que o grupo que treinou com velocidade de execução rápida, foi capaz de realizar maior volume total de carga durante a sessão de exercício, fato que resultou no tempo de recuperação da força muscular pós-sessão de exercício em 48h, comparado ao grupo que treinou com velocidade de execução lenta e necessitou de $24 \mathrm{~h}$ para o restabelecimento do desempenho neuromuscular.

Outro fator que devemos levar em consideração ao analisar esses estudos, seria o método utilizado para avaliar o desempenho neuromuscular pós-sessão de exercício. Mclester et al. ${ }^{5}$ analisaram o desempenho de uma sessão de exercício de força realizado em 3 séries de 10RM até a falha concêntrica em diferentes exercícios, incluindo o supi-

\section{REFERÊNCIAS}

1. Bird SP, Tarpenning KM, Marino FE. Designing resistance training programmes to enhance muscular fitness: a review of the acute programe. Sports Med. 2005;35(10):841-51.

2. American College of Sports Medicine. Progression models in resistance training for healthy adults. Med Sci Sports Exerc. 2009;41(3):687-708.

3. Smith LL. Tissue trauma: the underlying cause of overtraining syndrome? J Strength Cond Res. 2004;18(1):185-93.

4. De Souza RW, Aguiar AF, Carani FR, Campos GE, Padovani CR, Silva MD. High-intensity resistance training with insufficient recovery time between bouts induced atrophy and alterations in myosin heavy chain content in rat skeletal muscle. Anat Rec. (Hoboken) 2011;294(8):1393-400.

5. Mclaster JR, Bishop PA, Smith J, Wyers L, Dale B, Kozusko J, et al. A series of studies-a practical protocol for testing muscular endurance recovery. J Strength Cond Res. 2003;17(2):259-73.

6. Judge LW, Burk JR. The effect of recovery on strength performance following a high-intensity bench press workout in males and females. Int J Sports Physiol Perform. 2010;5(2):184-96.

7. Jones EJ, Bishop PA, Richardson MT, Smith JF. Stability of practical measure of recovery from resistance training. J Strength Cond Res. 2006;20(4):756-9.

8. Bishop PA, Jone E, Woods AK. Recovery from training: a brief review: brief review. J Strength Cond Res. 2008;22(3):1015-24.

9. Jentjens $R$, Jeukendrup A. Determinants of post-exercise glycogen synthesis during short-term recovery. Sports Med. 2003;33(2):117-44

10. Chargé SB, Rudnicki MA. Cellular and molecular regulationn of muscle regeneration. Physiol Rev. 2004;84(1):209-38 no. A recuperação neuromuscular (resistência de força) foi considerada no momento em que os indivíduos conseguiram replicar as três séries de 10RM até a falha concêntrica, pós-sessão. Judge e Burke ${ }^{8}$ estimaram a força máxima muscular (1RM), por meio do percentual equivalente para o número de repetições completadas na carga de 5RM no supino, enquanto que Lopes et al. ${ }^{16}$ determinaram a força máxima muscular por meio do teste tradicional de 1RM.

No presente estudo, foi utilizado acelerômetro (Myotest ${ }^{\circledR}$ ) para mensurar a capacidade neuromuscular (força, velocidade e potência) na fase concêntrica do movimento. Segundo Comstock et al. ${ }^{20}$, tal instrumento demonstrou alta confiabilidade e validade no exercício supino para determinar a força e potência, quando comparado com instrumentos padrão ouro (plataforma de força e transdutor linear). Dessa forma, destacamos que nossos dados foram coletados com ferramenta prática, porém validada, para avaliar indiretamente a capacidade neuromuscular.

\section{CONCLUSÃO}

Nossos resultados sugerem que o intervalo de recuperação de 24 horas foi suficiente para a recuperação da capacidade neuromuscular máxima (força, velocidade e potência) de forma significativa, em homens com experiência em treinamento de força. Desta forma, a programação de sessões de exercícios de força, com foco no aprimoramento das adaptações neurais, repetidas para o mesmo grupamento muscular (peitoral), pode ser realizada em dias seguidos (período de $24 \mathrm{~h}$ ) para indivíduos treinados, não interferindo no desempenho neuromuscular. No entanto, necessita-se da realização de estudos crônicos para a determinação do potencial adaptativo de indivíduos treinados, por meio da manipulação do intervalo de recuperação entre sessões.

\section{AGRADECIMENTOS}

Os autores agradecem aos voluntários que participaram do estudo e academia R Personal Training, pelo espaço cedido para a realização do estudo.

Todos os autores declararam não haver qualquer potencial conflito de interesses referente a este artigo.

11. Nosaka K, Aoki MS. Repeated bout effect: research update and future perspective. Braz J Biomotr. 2001;5(1):5-15.

12. Newton MJ, Morgan GT, Sacco P, Chapman DW, Nosaka K. Comparison of responses to strenuos eccentric exercise of the elbow flexors between resistance-trained and untrained men. J Strength Cond Res. 2008;22(2):597-607.

13. Häkkinen K. Neuromuscular fatigue and recovery in male and female athletes during heavy resistance exercise. Int J Sports Med. 1993;14(2):53-9.

14. Raastad T, Hallén J. Recovery of skeletal muscle contractility after high- and moderate-intensity strength exercise. Eur J Appl Physiol. 2000;82(3):206-14.

15. Ide BN, Leme TC, Lopes CR, Moreira A, Delchechi C, Serraipa MF, et al. Time course of strength and power recovery after resistance training with different movement velocities. J Strength Cond Res. 2011;25(7):2025-33.

16. Lopes CR, Crisp AH, Rodrigues AL, Teixeira AG, da Mota GR, Verlengia R. Fast contraction velocity in resistance exercises induced greater total volume load lifted and muscle strength loss in resistance-trained men. Rev Andal Med Desporte. 2012;5(4):123-6.

17. Brown LE, Weir JP. ASEP - Procedures recommendation I: accurate assessment of muscular strength and power. J Exerc Physiol. 2001;4(1):1-21

18. Koshida S, Urabe Y, Miyashita K, Iwai K, Kagimori A. Muscular outputs during dynamic bench press under stable versus unstable conditions. J Strength Cond Res. 2008;22(5):1584-8.

19. Falvo MJ, Schilling BK, Bloomer RJ, Smith WA. Repeated bout effect is absent in resistance trained men: an electromyographic analysis. J Electromyogr Kinesiol. 2009;19(6):e529-35.

20. Comstock BA, Solomon-Hill G, Flanagan SD, Earp JE, Luk HY, Dobbins KA, et al. Vality of the Myotest ${ }^{\oplus}$ in measuring force and power production in the squat and bench press. J Strength Cond Res. 2011;25(8):2293-7.

\section{ERRATA}

No artigo intitulado "INDICADORES ANTROPOMÉTRICOS DE ESTADO NUTRICIONAL COMO PREDITOR DE CAPACIDADE EM IDOSOS", de autoria de Kleyton Trindade Santos, José Carlos Cândido dos Santos Júnior, Saulo Vasconcelos Rocha, Luciana Araújo dos Reis, Raildo da Silva Coqueiro, Marcos Henrique Fernandes, publicado na Revista Brasileira de Medicina do Esporte (RBME) vol. 20, n 3, 2014, páginas 181-5, DOI: http://dx.doi.org/10.1590/1517-86922014200301816, por solicitação dos autores, onde se apresentava os autores RAILDO DA SILVA COQUEIRO e SAULO VASCONCELOS ROCHA como afiliados à Universidade Federal do Rio Grande do Norte, o correto é que são afiliados à Universidade Estadual do Sudoeste da Bahia. 